\title{
Radiation safety summit: nuclear medicine and PET/CT justification and optimization
}

\author{
Helen R. Nadel
}

(C) Springer-Verlag 2011

Widespread concern about radiation exposure requires a serious review of justification and protocols for all imaging studies including dedicated scintigraphic procedures in nuclear medicine as well as hybrid procedures for both $\mathrm{PET} / \mathrm{CT}$ and SPECT/CT. Consultation with the imaging specialist who will be performing and interpreting the test should include an appropriate review of all of the patient's relevant imaging prior to the requested study. This will ensure that the requested study is indeed justified. After it is determined the study will add to care of the child, the scintigraphic or hybrid study to be undertaken should be tailored to the particular clinical question to be answered. Repeating studies is to be avoided.

Adhering to guidelines for administered radiopharmaceutical doses in children should optimize pediatric nuclear medicine studies. There are two published standards for mainly weight-based pediatric radiopharmaceutical dose administration: (1) Consensus guidelines for administered radiopharmaceutical activities in children and adolescents by Gelfand et al., recently published in the Journal of Nuclear Medicine; and (2) the European Association of Nuclear Medicine (EANM) pediatric dose card by Lassmann et al., published in 2007 in the European Journal of Nuclear Medicine and Molecular Imaging. Certain radiopharmaceuticals are not recommended for use in children. Indium-111 for inflammation has generally not been utilized in the pediatric age group because of possible damage to lymphocytes. More recently the North American

Disclaimer Dr. Nadel has no financial interests, investigational or offlabel uses to disclose.

H. R. Nadel $(\bowtie)$

BC Children's Hospital,

Vancouver, Canada

e-mail: hnadel@cw.bc.ca consensus guidelines recommend that gallium-67 should only be used infrequently in children and only in low doses because of the high absorbed radiation dose from this radiopharmaceutical. The more widespread use of PET/CT and SPECT/CT hybrid imaging has replaced many of the indications for gallium scintigraphy. Additional reduction of radiation exposure in general nuclear medicine might be possible with software resolution recovery programs to improve image resolution, if lower administered radiopharmaceutical dose is utilized with longer acquisition time.

In hybrid imaging to include PET/CT and SPECT/CT, the focus has been on how to reduce the CT dose and adhere to the ALARA principle. Many organizations are providing guidelines and information on the risks of CT examinations in children and information on pediatricspecific CT protocols that should be utilized when performing CT examinations in children (Table 1). For both SPECT/CT and PET/CT the acquisition parameters and DLP and CTDI recorded at the scanner at the time of the study should become part of the permanent record and sent to the PACS. There are now software programs that can calculate the patient effective dose on the fly as the study is performed or retrospectively if these data are available. An accurate cumulative measure of patient effective dose should be part of the patient's health record as we enter the era of the electronic health record.

SPECT/CT studies are mainly performed for tumor studies utilizing I-123 metaiodobenzylguanidine (MIBG) or for bone imaging. In these studies it is particularly important for the imaging physician to tailor the examination. At the time of the request for examination it should be determined whether a CT in addition to the SPECT study might be warranted. If the child has recently had a crosssectional imaging study (CT or MR) that could be fused with post-processing fusion software, then an additional CT 
Table 1 Radiation safety sites

\begin{tabular}{ll}
\hline $\begin{array}{l}\text { Image } \\
\text { Gently }\end{array}$ & www.imagegently.org \\
NCI & www.cancer.gov/cancertopics/causes/radiation-risks- \\
& pediatric-CT \\
ACR & www.acr.org/secondarymainmenucategories/ \\
& quality_safety/radsafety \\
\hline
\end{tabular}

might not be warranted. Current computer programs can often adequately fuse outside studies in DICOM format. If a subsequent MR study is to be undertaken for the patient, then exposing the child to the radiation for the CT part of the SPECT/CT study might not be warranted, as again the MR and SPECT study can often be evaluated with image fusion. In hybrid machines capable of performing diagnostic-quality scans, axial coverage of the CT should be determined by the clinical question or by the findings on the SPECT portion of the study. All of the current SPECT/ CT scanners can perform the CT portion of the study temporally after the SPECT acquisition.

In bone scintigraphy or for localization studies only, the axial field of view of the CT examination can be limited to a field of view smaller than the SPECT acquisition to provide required diagnostic information of the SPECT abnormality. In these hybrid bone studies it is also possible to markedly reduce the $\mathrm{kVp}$ and $\mathrm{mAs}$ of the CT acquisition, as the fusion to the scintigraphic abnormality will aid in localization of the abnormality and the characterization does not require high-dose or high-resolution CT studies in most cases.

In hybrid scanners capable of performing diagnosticquality CT studies, the CT scan can suffice as the single diagnostic study if intravenous contrast agent is utilized, thereby obviating the need for an additional stand-alone CT scan. We utilize this technique for patients requiring both MIBG scintigraphy and CT examination (Fig. 1). Performing a single MIBG SPECT/CT study will also eliminate another sedation that might be required if the child were to have an additional stand-alone CT scan. These CT studies can be performed as helically acquired breathhold studies with reconstruction for different tissue types and slice thickness exactly the same as stand-alone CT. All standard CT post-processing capabilities including 3-D multiplanar reformatting are possible on hybrid scanners that have diagnostic capability. Dose modulation programs are readily available for the hybrid scanners and should be utilized for all CT studies that are performed, further incurring radiation savings.

For PET/CT studies, a best-practice scenario would be to perform the CT for the hybrid procedures as one would perform a stand-alone CT. A single IV-contrast-enhanced CT scan performed at the time of PET/CT can suffice for both diagnostic localization and attenuation correction without introduction of significant artifacts to either study that might hamper diagnostic evaluation. The use of iodinated contrast agent and a lower $\mathrm{kV}$ is the basis for dual-energy CT advances. In addition, the CT scan can be performed with lower mAs dose technique given the paradigm shift of the importance of the metabolic information obtained from the PET examination. Lowering $\mathrm{kVp}$ can reduce radiation dose by $25-50 \%$ without affecting image quality, and there is a linear relationship between $\mathrm{mAs}$ reduction and dose reduction. Parameters of $80 \mathrm{kVp}$ and 40-60 mAs can provide good diagnostic information and reduce dose of the $\mathrm{PET} / \mathrm{CT}$ from approximately $21 \mathrm{mSv}$ to $7-9 \mathrm{mSv}$. The use of dose modulation will further reduce the radiation dose (Fig. 2).

Technical advances are also occurring on the CT side of these hybrid scanners to include dual energy acquisition, adaptive $\mathrm{kVp}$ settings during the scan, and iterative reconstruction techniques that might allow additional dose reduction. One limitation of a single optimized PET/CT study has been the inability to optimize lung visualization for detection of pulmonary nodules. Newer

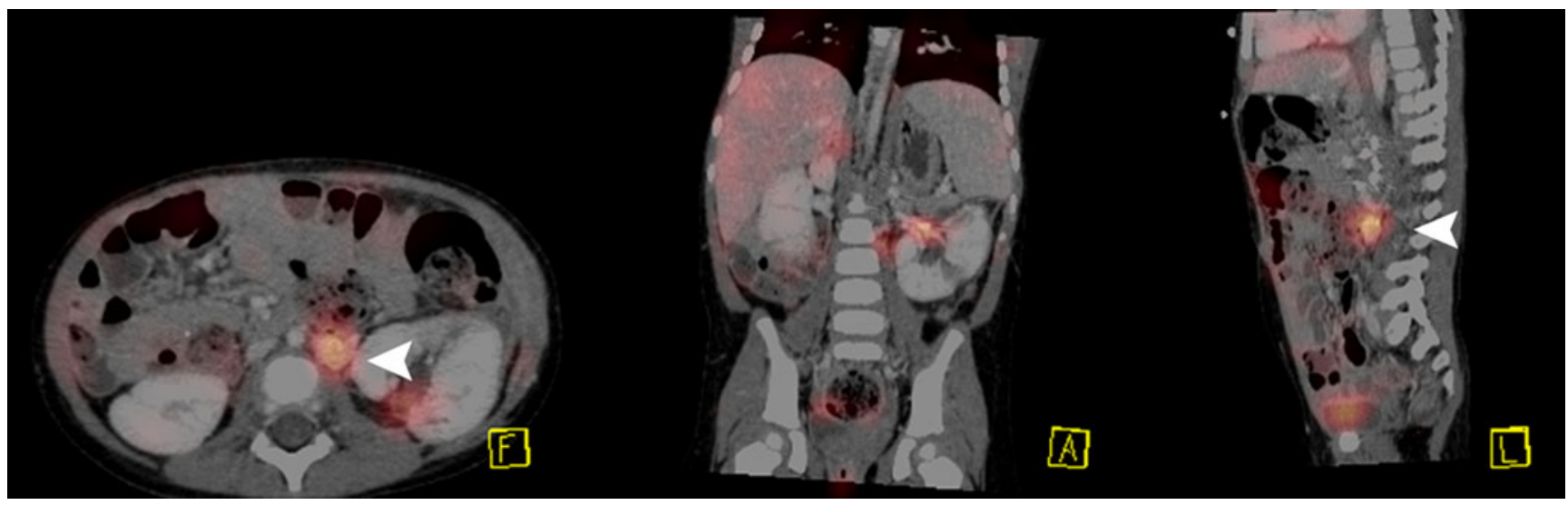

Fig. 1 In a child with neuroblastoma, the diagnostic post-intravenous-contrast-enhanced I-123 MIBG SPECT/CT scan identifies a small focus of residual disease (white arrowhead) in a para-aortic location 


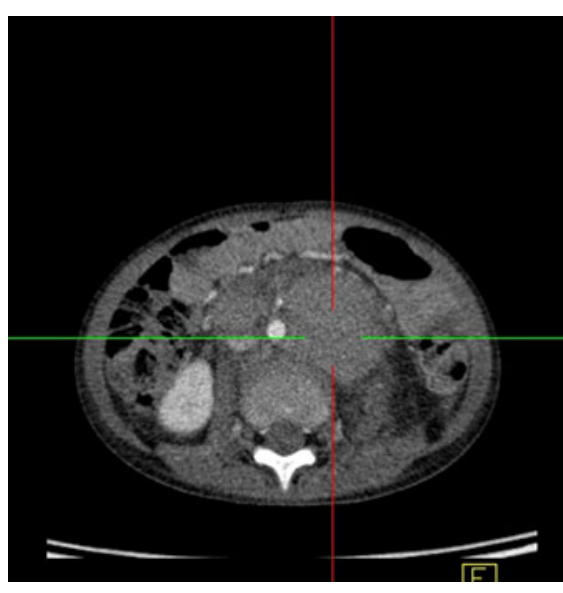

Fig. 2 Staging 18-F FDG (fluorodeoxyglucose) PET/CT study performed as an optimized post-intravenous-contrast-enhanced PET/ CT scan in a child with lymphoma shows extensive retroperitoneal

PET/CT scanners all provide respiratory gating and many can tailor the acquisition of different parts of the examination, e.g., a breath-hold study through the thorax could be seamlessly integrated into the PET/CT acquisition. If such equipment is not possible then a stand-alone breath-hold study of the lungs is still recommended where the child is at risk of pulmonary metastatic disease. Most common practice is to do a CT scan for attenuation purposes of the PET study, with very low $\mathrm{mAs}$ and pediatric-recommended $\mathrm{kVp}$, and to perform a diagnostic-quality post-contrast-enhanced $\mathrm{CT}$ scan as a

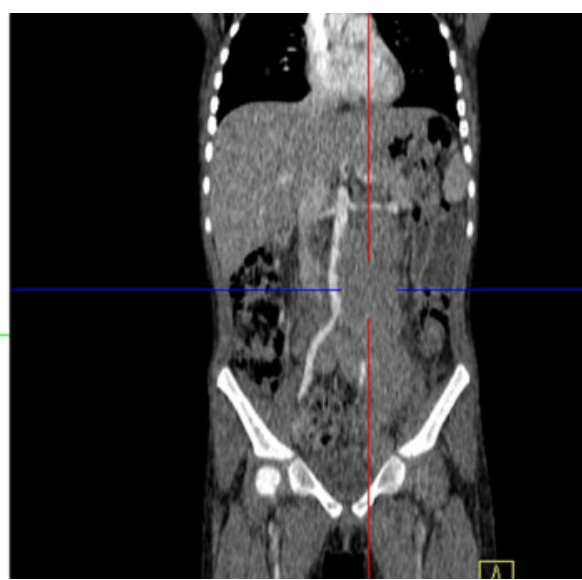

adenopathy. CT acquisition parameters were $80 \mathrm{kVp}$ and $40 \mathrm{mAs}$. With dose modulation, the mAs recorded in the mid-abdomen was $25 \mathrm{mAs}$

stand-alone study. This practice of not performing a single optimized study might have more to do with economic reimbursement factors and "turf" battles resulting from the separation of nuclear medicine and radiology for reporting purposes. Not performing a second $\mathrm{CT}$ for scintigraphic functional imaging studies such as PET/CT must be seen as a definite positive step. Efforts to change the dichotomy of radiology and nuclear medicine and further our collaboration must become a top priority to further reducing exposure to our pediatric patients as hybrid imaging techniques advance. 